\title{
Combate à corrupção através de relacionamentos interorganizacionais transparentes
}

\author{
Bruna Diirr ${ }^{1}$, Claudia Cappelli ${ }^{1}$ \\ ${ }^{1}$ Programa de Pós-Graduação em Informática - Universidade Federal do Estado do Rio \\ de Janeiro (UNIRIO) \\ Rio de Janeiro - RJ - Brasil \\ \{bruna.diirr, claudia.cappelli\} duniriotec.br
}

\begin{abstract}
Organizations increasingly need to establish partnerships to face environment changes and remain competitive. Resources sharing enables them to better handle the identified opportunity for joint work. Such associations are also find at governmental level. However, the interorganizational relationships management faces many challenges. This paper aims to explore this topic, especially regarding public transparency. It is proposed an approach that intends to make partner organizations more integrated, prepared to interoperate processes and information and able to achieve shared goals even with existing differences. An anti-corruption partnership illustrates the approach application.
\end{abstract}

Resumo. Cada vez mais, as organizações precisam estabelecer parcerias para enfrentarem mudanças no ambiente em que atuam e permanecerem competitivas. O compartilhamento de recursos permite que essas organizações lidem melhor com a oportunidade identificada para colaboração. Tais associações também estão presentes no âmbito governamental. Porém, a gestão de relacionamentos interorganizacionais apresenta muitos desafios. Este artigo tem como objetivo explorar esse assunto, especialmente no que diz respeito à transparência pública. É apresentada uma abordagem que visa tornar as organizações parceiras mais integradas, preparadas para interoperar processos e informações e com habilidades para alcançar objetivos compartilhados mesmo com as diferenças existentes. Uma parceria para combate à corrupção ilustra a aplicação da abordagem.

\section{Introdução}

Transformações na economia, globalização, inovações tecnológicas, rápida difusão da informação, demandas para redução do tempo e custo do ciclo de desenvolvimento, e necessidade de fornecer mais transparência a processos e informações existentes, levaram à mudança e adaptação das organizações para continuarem competitivas. Uma nova abordagem das organizações tem sido investir no estabelecimento de parcerias com outras organizações, rivais ou operando em segmento distinto aos seus, para enfrentar um ambiente cada vez mais dinâmico, imprevisível, altamente competitivo e desafiador [Corso e Fossá 2009][Fusco 2005][Mueller et al 2013][Ranaei et al 2010][Van Fenema et al 2014]. Em geral, essas organizações selecionam um tipo específico de relacionamento, como alianças, redes, joint ventures, terceirizações etc., 
levando em consideração a oportunidade identificada para o trabalho conjunto, interesses comuns, confiança decorrente da transparência existente entre os envolvidos, realidade enfrentada, estrutura existente e acordos estabelecidos, de forma a colaborar para alcançar um objetivo comum. Esta colaboração permite que as organizações tenham acesso a um leque mais amplo de ferramentas do que as que elas próprias possuem, respondam mais rapidamente aos desafios do mercado através da complementação de competências existentes e compartilhem os resultados alcançados através da parceria [Corso e Fossá 2009][Del-Río-Ortega et al 2015][Fusco 2005] [Khalfallah 2013][Ranaei et al 2010][Sebu e Ciocârlie 2015][Van Fenema et al 2014].

Esse investimento em relacionamentos interorganizacionais não está restrito às organizações que visam se manter competitivas em um ambiente cada vez mais desafiador. É algo que também ocorre no âmbito governamental. Em várias ações brasileiras anticorrupção [El País 2016][Estadão 2017][G1 2016][Polícia Federal 2017], é possível notar a articulação de diferentes agências governamentais. Por serem responsáveis por alguns aspectos da investigação, seja em termos de alçada de atuação ou de acesso à informação requerida, elas precisam colaborar para que os envolvidos sejam identificados e punidos.

Contudo, apesar de todos os benefícios obtidos com os relacionamentos interorganizacionais, essa nova dinâmica de atuação estimula a interação de organizações com diferentes características, culturas e valores. Isso aumenta a probabilidade de serem enfrentados mal-entendidos e conflitos, influenciando assim o alinhamento da atuação desses parceiros. As organizações parceiras precisam desenvolver habilidades para trabalharem nessa nova dinâmica, identificando, integrando e gerenciando todos os elementos compartilhados de forma a garantir que eles favoreçam a execução das atividades que apoiam a integração da estratégia do grupo e permitam atingir seus objetivos. Se não for possível estabelecer um meio-termo entre a variedade de dinâmicas existentes, o relacionamento interorganizacional pode fracassar e até levar à dissolução da parceria estabelecida [Bocanegra et al 2011][Corso e Fossá 2009][Pedroso 2009][Sebu e Ciocârlie 2015][Van Fenema et al 2014][Zhu e Huang 2007].

O presente artigo visa explorar o desafio de gerir relacionamentos interorganizacionais, especialmente os desafios relacionados à transparência pública. Para isso, é detalhada uma abordagem baseada em objetivos compartilhados. Argumenta-se que, assim, as organizações envolvidas estão mais integradas, transparentes, preparadas para interoperar seus processos e informações e capazes de agir e alcançar um objetivo compartilhado mesmo com as diferenças existentes. Essa abordagem é aplicada a uma parceria formada para combate à corrupção e criticada de forma a guiar o trabalho futuro.

O artigo está organizado da seguinte forma: a seção 2 caracteriza a dinâmica de combate à corrupção, discutindo também artigos relacionados à gestão de relacionamentos interorganizacionais. A seção 3 descreve a abordagem proposta para gestão de relacionamentos interorganizacionais, discutindo a importância da transparência durante essa gestão. A seção 4 ilustra a aplicação da abordagem em uma parceria governamental para combate à corrupção. Por fim, na seção 5, são expostas as conclusões obtidas com o desenvolvimento deste trabalho. 


\section{Relacionamentos interorganizacionais para combate à corrupção}

De acordo com recente avaliação, a organização Transparency International (2017) afirma que a corrupção continua a ser uma "praga" em todo o mundo, tornando evidente que a sociedade já não tolera mais estas ações e demanda que o problema seja enfrentado. O Brasil não aparece bem neste cenário, obtendo 40 pontos (de 0 , altamente corrupto, a 100, muito limpo) e ocupando assim o $79^{\circ}$ lugar de 176 países avaliados. Esta organização ainda afirma que os países com melhor desempenho compartilham características chave como acesso à informação e participação do cidadão. $\mathrm{O}$ acesso à informação permite criar uma sociedade democrática com cidadãos participativos, fornecendo-lhes ferramentas para compreender e utilizar a informação e estimulando um pensamento crítico sobre as informações e serviços prestados. A Lei de Acesso à Informação [Brasil 2009][Brasil 2011] tem incentivado a transparência ativa, o que propiciou uma maior disponibilidade de informação sobre as organizações públicas em seus sites institucionais. Essa disponibilização de informações possibilita a identificação de casos de corrupção pela própria Sociedade, como o caso mais recente envolvendo desvios de bolsas da UFPR [Gazeta do Povo 2017].

O combate à corrupção, muitas vezes, demanda articulação de diferentes agências governamentais. Por serem responsáveis por determinados aspectos da investigação, elas precisam colaborar para que os envolvidos sejam identificados e punidos. Assim, essas agências podem compartilhar recursos humanos e financeiros, sistemas, equipamentos, processos e informações existentes, fazendo com que elas tenham acesso a maior ferramental com custo mais favorável do que conseguiriam sozinhas. Elas também conseguem organizar informações que muitas vezes são relacionadas, mas como cada informação reside em uma agência diferente, ficam espalhadas em bases de dados isoladas. Além disso, elas conseguem responder aos desafios identificados mais rapidamente devido à complementação das habilidades existentes [Corso e Fossá 2009][Ranaei et al 2010].

Porém, tais agências precisam estar preparadas para enfrentar uma nova dinâmica de trabalho. Ao atuarem sozinhas, cada agência somente precisa gerir seu pessoal, processos, informações, sistemas, equipamentos e recursos financeiros para atingir os objetivos. $\mathrm{O}$ estabelecimento de relacionamentos interorganizacionais estimula a interação de pessoas com diferentes características, culturas e valores, influenciando assim o alinhamento da atuação das agências envolvidas [Corso e Fossá 2009][Zhu e Huang 2007]. Isso aumenta a probabilidade de serem enfrentados malentendidos e conflitos, uma vez que as agências possuem distintas formas de planejamento, tomadas de decisão, alocação de recursos e definição de processos e informações. Se não for possível estabelecer um meio-termo entre a variedade de dinâmicas existentes, com envolvidos enfrentando problemas no relacionamento e surgimento de conflitos, o relacionamento interorganizacional pode fracassar, levando a um enorme prejuízo para a Sociedade [Corso e Fossá 2009][Sebu e Ciocârlie 2015].

Dessa forma, para um trabalho conjunto eficaz, assegurando que os elementos existentes favoreçam a execução de atividades que apoiem o combate à corrupção, é necessário minimizar as diferenças de pensamentos e estilos de trabalho. As agências parceiras precisam identificar, integrar e gerir todos os elementos que compartilham, desenvolvendo habilidades que permitam lidar com as diferenças existentes de modo 
que o trabalho conjunto seja eficaz, os objetivos compartilhados sejam alcançados e o relacionamento possa ser considerado de sucesso [Bocanegra et al 2011][Corso e Fossá 2009][Denis et al 2017][Pedroso 2009][Zhu e Huang 2007]. Contudo, fazer com que agências estejam integradas e tenham habilidades para conviver com diferenças não é uma tarefa trivial. São necessários métodos, técnicas e ferramentas adequadas a isso.

Autores argumentam que os membros das organizações são considerados elementos chave para o alinhamento interorganizacional, sendo preciso estimular seu comprometimento com os objetivos compartilhados, conscientização das diferenças e aprendizado sobre o comportamento em uma dinâmica organizacional nova, de forma a garantir a efetividade do trabalho conjunto [Bocanegra et al 2011][Corso e Fossá 2009] [Pedroso 2009][Zhu e Huang 2007]. A Arquitetura Empresarial é outro aspecto importante, com as organizações parceiras precisando explicitar como organizar o fluxo de informações, facilitar a troca de conhecimento e manter direitos sobre propriedade intelectual, de forma a entender como cada uma delas pode melhor contribuir para a parceria, definir acordos para utilização dos recursos disponíveis e melhor integrar os mesmos [Choi e Kröschel 2015][Drews e Schirmer 2014][Mueller et al 2013][Nunes et al 2016]. A compreensão do que se quer alcançar com o relacionamento interorganizacional e o que se pode fazer também é necessária, com demandas de melhoria e extensão das metodologias para modelagem do negócio e dos objetivos compartilhados de forma a lidar com a complexidade e particularidades dos relacionamentos interorganizacionais [Bouchbout e Alimazighi 2011][Lawall et al 2014][Sebu e Ciocârlie 2015]. Além disso, é necessário refletir sobre os sistemas adotados pelas organizações participantes (Sistemas de Informação Interorganizacionais) para que eles apoiem a parceria, não sejam impactados pelas diferenças existentes e estejam alinhados com a estratégia de negócio [Hsu et al 2015] [Kauremaa e Tanskanen 2016][Sun e Yu 2011].

Embora descrevam propostas que poderiam ajudar as organizações envolvidas em parcerias, os estudos citados estão interessados somente em algum aspecto da gestão de relacionamentos interorganizacionais, negligenciando também o aspecto de transparência. As organizações parceiras precisam desenvolver habilidades para trabalharem nessa nova dinâmica, identificando, integrando e gerindo todos os elementos compartilhados de forma a garantir que eles favoreçam a execução de atividades que apoiam a integração da estratégia de grupo e alcance dos objetivos compartilhados. Mecanismos que tornem as organizações envolvidas mais integradas, transparentes, preparadas para interoperar seus processos e informações e capazes de agir e alcançar os objetivos compartilhados ainda se fazem necessários.

\section{Gestão de relacionamentos interorganizacionais baseada em objetivos compartilhados}

A abordagem proposta para gestão de relacionamentos interorganizacionais utiliza os objetivos compartilhados para a integração das organizações parceiras (Figura 1). Argumenta-se que o objetivo é elemento comum entre as organizações que se tornam parceiras, sendo possível elencar os demais aspectos necessários à parceria a partir dele. 


\begin{tabular}{|c|c|c|c|c|c|}
\hline $\begin{array}{c}\text { Entender } \\
\text { oportunidade } \\
\text { O que temos que fazer? }\end{array}$ & $\begin{array}{l}\text { Detalhar insumos } \\
\text { necessários } \\
\text { Como devemos fazer? }\end{array}$ & $\begin{array}{c}\text { Mapear insumos } \\
\text { existentes em cada } \\
\text { organização } \\
\text { O que sei fazer? }\end{array}$ & $\begin{array}{l}\text { Identificar insumos } \\
\text { existentes na parceria } \\
\text { O que conseguimos fozer } \\
\text { juntos? }\end{array}$ & $\begin{array}{l}\text { Definir processos da } \\
\text { parceria } \\
\text { Como vamos fazer? }\end{array}$ & $\begin{array}{c}\text { Atribuir } \\
\text { responsabilidades } \\
\text { Quem vai fazer? }\end{array}$ \\
\hline
\end{tabular}

Figura 1. Abordagem para gestão de relacionamentos interorganizacionais baseada em objetivos compartilhados

Assim, primeiramente é necessário entender a oportunidade que gerou a atuação conjunta das organizações, determinando o objetivo compartilhado entre elas. É importante destacar que tal objetivo pode ser desafiador e complexo, sendo necessário detalhá-lo em diferentes subobjetivos e explicitar a relação entre eles. A partir disso, é possível detalhar como a atuação da parceria precisa ocorrer. Isso envolve a identificação de processos, papéis, informações, sistemas, equipamentos e habilidades necessários, considerando as regras que os delimitam (Figura 2).

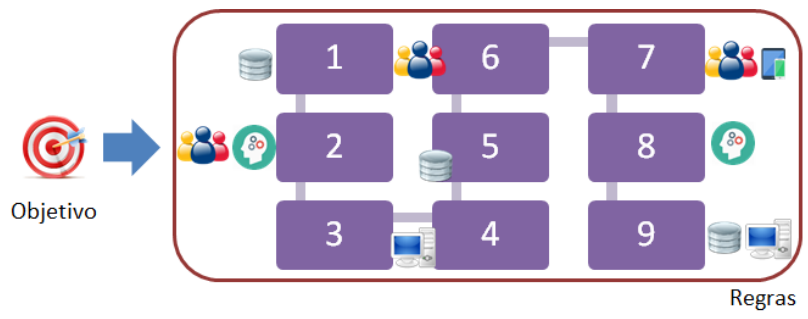

Figura 2. Insumos necessários para atingir o objetivo compartilhado

O próximo passo é mapear o que cada integrante da parceria pode fornecer para atingir o objetivo compartilhado. Isso envolve a (a) caracterização de cultura, (b) identificação de habilidades existentes e (c) identificação de processos, informações, sistemas, tecnologia e recursos financeiros de cada uma das organizações envolvidas. A Figura 3 apresenta como cada organização poderia apoiar o mapeamento apresentado na Figura 2.

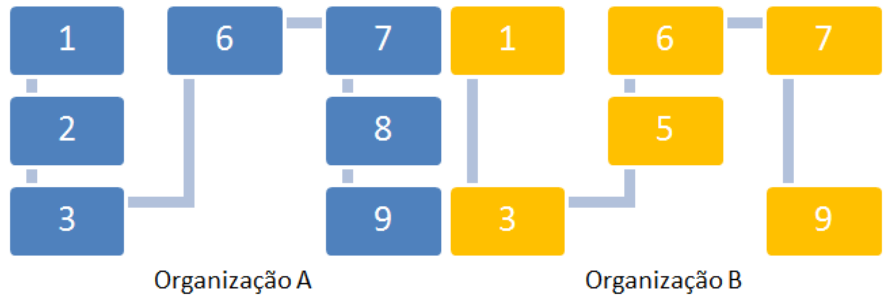

Figura 3. Insumos disponíveis nas organizações

Conhecendo as organizações participantes, é possível identificar os insumos existentes na parceria (Figura 4). Isso é possível através do cruzamento entre o mapeamento de cada uma das organizações parceiras (Figura 3) e o detalhamento de como a atuação dessas organizações precisa ocorrer para atingir o objetivo compartilhado (Figura 2). Os processos da parceria também devem ser definidos. Caso a parceria não possua algum dos insumos necessários e de acordo com os aspectos relacionados, é necessário: (a) processo: buscar outra organização para participar da parceria, buscar capacitação externa, desenvolver o processo requerido ou mesmo substituir tal processo; (b) pessoal: contratar pessoal ou capacitar pessoal não envolvido; (c) sistema: adquirir ou desenvolver sistema; (d) informação: gerar informação ou buscar informação externamente; (e) tecnologia: adquirir ou alugar equipamento. 


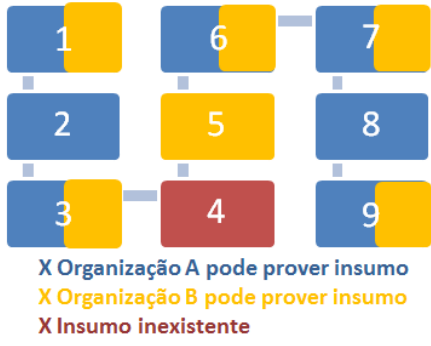

Figura 4. Insumos disponíveis na parceria

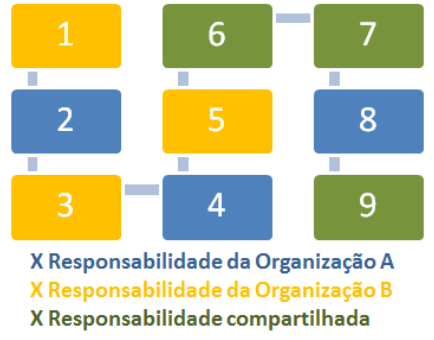

Figura 5. Atribuição de responsabilidades

Por fim, a responsabilidade de cada organização envolvida na parceria é atribuída (Figura 5), identificando o que é de responsabilidade de cada parceiro e o que está sob responsabilidade compartilhada. Negociações devem existir quando mais de um parceiro for capaz de fornecer um mesmo insumo. Para atividades compartilhadas, será preciso integrar culturas, processos e informações.

Argumenta-se que tal abordagem permite que as organizações envolvidas se tornem mais integradas, preparadas para interoperar seus processos e informações e capazes de agir e alcançar os objetivos compartilhados. Quando aplicada no âmbito governamental, também poderia prover transparência organizacional, adicionando valores sociais relacionados com características como auditabilidade, acessibilidade, compreensão, clareza, confiabilidade etc. [Cappelli et al 2014][Denis et al 2017].

\section{Aplicação da abordagem no combate à corrupção}

Muito se tem noticiado sobre a atuação da Polícia Federal em investigações de combate à corrupção [Polícia Federal 2017]. Ela é a responsável por investigar atos criminais contra a ordem política e social ou em detrimento de interesses do governo federal, bem como infrações com repercussões interestaduais ou internacionais. De acordo com o rumo da investigação, faz-se necessária sua associação com outras agências governamentais. Em relação à apropriação indevida de dinheiro público, a Receita Federal deve ser envolvida. Esta agência é responsável pela administração de impostos federais, combate à evasão fiscal e atos ilegais relacionados ao comércio internacional (contrabando, pirataria, tráfico etc.).

Dado este contexto, o primeiro passo para a aplicação da abordagem proposta é entender a oportunidade que gerou a colaboração das agências. Assim, o objetivo compartilhado é combater a corrupção, identificando, investigando e punindo pessoas e/ou organizações que se apropriaram indevidamente de dinheiro público. A partir dele, os insumos para a operação da parceria são detalhados. Durante o combate à corrupção, agentes da Polícia Federal e da Receita Federal realizam um conjunto de atividades que requerem informações, capturadas da base de dados da Polícia Federal, declaração de imposto de renda, operações bancárias, escutas telefônicas autorizadas, ordens judiciais etc.; uso de sistemas da Polícia Federal e da Receita Federal; equipamentos como veículos, armas etc.; e deve seguir as regras definidas nas leis brasileiras (Figura 6). 


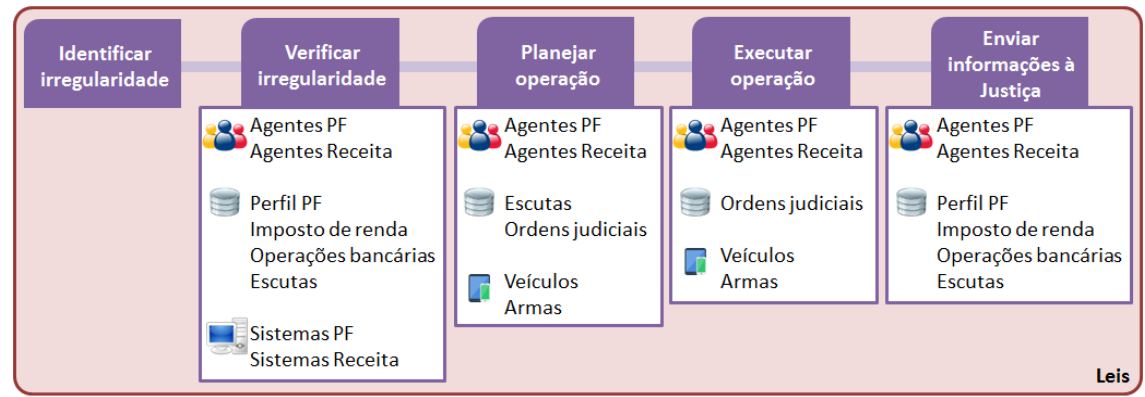

Figura 6. Insumos necessários para o combate à corrupção

A partir desse detalhamento, é possível mapear o que Polícia Federal e Receita Federal podem prover ao combate à corrupção, sendo possível identificar os insumos disponíveis nessa parceria (Figura 7). Por exemplo, a Polícia Federal (azul) pode fornecer agentes, informações extraídas de sua base de dados e escutas telefônicas autorizadas, sistemas existentes, veículos e armas. Já a Receita Federal (amarelo) pode fornecer agentes, informações do imposto de renda, sistemas existentes e veículos. Porém, essas agências não podem fornecer alguns dos insumos necessários (vermelho), como operações bancárias e ordens judiciais. Assim, é necessário obter essas informações externamente junto a agências financeiras e Justiça, respectivamente.

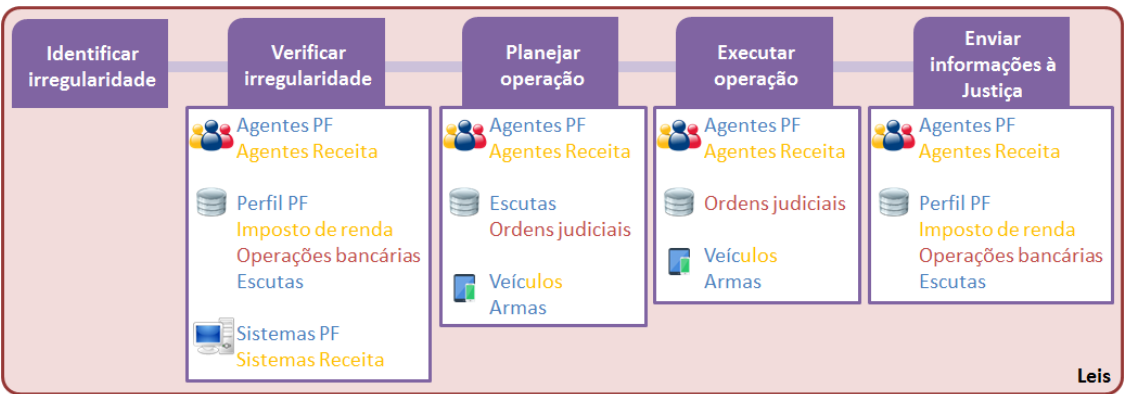

Figura 7. Insumos disponíveis na parceria para o combate à corrupção

Por fim, é necessário atribuir responsabilidades às agências envolvidas (Figura 8). Considerando a natureza das atividades, a Receita Federal será responsável pela verificação da irregularidade, uma vez que esta agência administra os impostos federais. Já a Polícia Federal será responsável pela execução da operação, pois possui em suas atribuições o cumprimento de ordens judiciais, e envio de informações à Justiça. Ambas agências serão responsáveis pela identificação de irregularidades, seja por investigação interna ou denúncias da Sociedade, além do planejamento da operação.

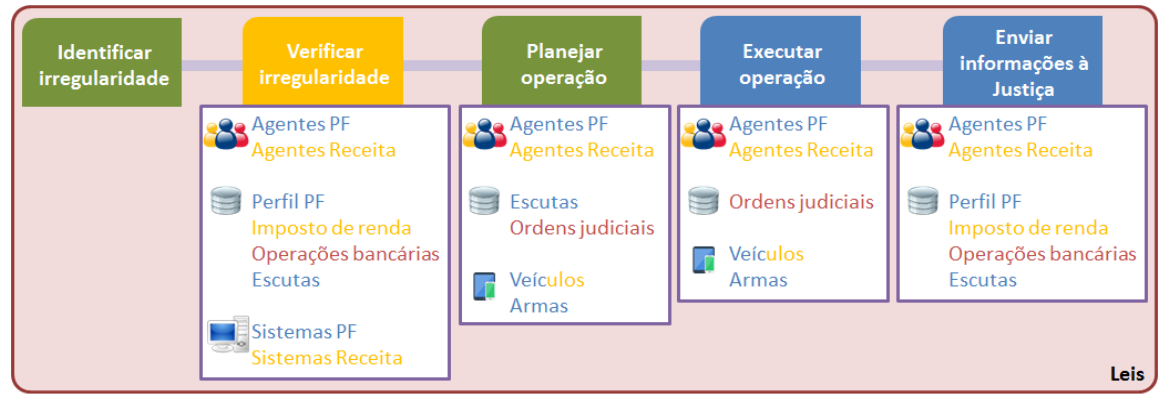

Figura 8. Atribuição de responsabilidades para o combate à corrupção 


\section{Conclusão}

A necessidade de continuarem competitivas em um ambiente cada vez mais dinâmico, imprevisível e desafiador leva as organizações a estabelecerem parcerias para combinar recursos, conhecimento e poder em benefício das organizações participantes. Tal necessidade também cresce cada vez mais no âmbito governamental, com diferentes agências interagindo em prol dos interesses da Sociedade. Contudo, se torna um desafio fazer com que distintas organizações se integrem e trabalhem em uma dinâmica nova, garantindo assim que o trabalho conjunto seja eficaz e bem-sucedido.

Este artigo explorou o desafio de gerir relacionamentos interorganizacionais, especialmente os desafios relacionados à transparência pública. Para isso, descreveu uma abordagem de apoio à gestão de relacionamentos interorganizacionais orientada aos objetivos compartilhados. Argumenta-se que essa abordagem torna as organizações parceiras mais integradas, transparentes, preparadas para interoperar seus processos e informações e capazes de agir e alcançar objetivos mesmo com as diferenças existentes.

A parceria entre agências para combate à corrupção ilustrou a aplicação da abordagem. Ele permitiu explorar a gestão de relacionamentos interorganizacionais, além de oferecer uma possibilidade para diferentes agências planejarem e organizarem seu trabalho conjunto para alcançar um objetivo compartilhado de forma transparente.

Contudo, é importante destacar alguns desafios que ainda precisam ser superados. O primeiro deles diz respeito ao detalhamento do objetivo. A descrição do objetivo compartilhado, além dos subobjetivos decorrentes dele, pode não ser tão clara para os parceiros. Também é possível lidar com parceiros que, apesar de saberem atuar em dadas circunstâncias, podem não ter processos internos descritos de uma forma estruturada. Além disso, as organizações podem ter dificuldades em fornecer os insumos inexistentes na parceria porque não conseguem identificar uma forma de fazer isso. Finalmente, a negociação para atribuição de responsabilidades pode ser árdua.

Como trabalhos futuros, a especificação de um protótipo de apoio à abordagem está em desenvolvimento. Além disso, visa-se avaliar a abordagem proposta em um cenário real, além de aplicá-la em outros domínios que não o governamental. Argumenta-se que os resultados obtidos fornecerão insumos para uma melhor compreensão do impacto da aplicação da abordagem e ajudarão na sua melhoria e evolução.

\section{Agradecimentos}

Os autores gostariam de agradecer à CAPES pelo financiamento que apoia a pesquisa.

\section{Referências}

Bocanegra, J.; Pena, J.; Ruiz-Cortes, A. (2011) "Interorganizational Business Modeling: An Approach for Traceability of Goals, Organizational Models and Business Processes", IEEE Latin America Transactions, v. 9, n. 1, pp.847-854.

Bouchbout, K.; Alimazighi, Z. (2011) "Inter-Organizational Business Processes Modelling Framework", In Conference on Advances in Databases and Information Systems, Vienna. 
Brasil (2009) Lei complementar $\mathrm{n}^{0} 131$ - Disponibilização em tempo real de informações, https://www.planalto.gov.br/ccivil_03/Leis/LCP/Lcp131.htm

Brasil. (2011) Lei $n^{\circ} 12.527$ - Lei de acesso à informação, http://www.planalto.gov.br/ ccivil_03/_ato2011-2014/2011/lei/112527.htm

Cappelli, C.; Engiel, P.; Leite, J. C.; Nunes, V.; Tanaka, A.; Santos, G.; Benjamim, K. (2014) "A Evolução do Modelo de Maturidade em Transparência Organizacional”, In Workshop de Transparência em Sistemas, Londrina.

Choi, T.; Kröschel, I. (2015) "Challenges of governing inter-organizational relationships: Insights from a case study", In European Conference on Information Systems, pp. 1-16, Münster.

Corso, K.; Fossá, M. I. (2008) "Discutindo as diferenças culturais, conflitos e os mecanismos de grupo em redes de empresas", In Encontro Nacional De Engenharia De Produção, Rio de Janeiro.

Denis, J. A.; Nunes, V.; Ralha, C.; Cappelli, C. (2017) "E-gov Transparency Implementation Using Multi-agent System: a Brazilian Study-Case in Lawsuit Distribution Process", In Hawaii International Conference on System Sciences.

Del-Río-Ortega, A.; Gutiérrez, A. M.; Durán, A.; Resinas, M.; Ruiz-Cortés, A. (2015) "Modelling Service Level Agreements for Business Process Outsourcing Services", In International Conference on Advanced Information Systems Engineering, LNCS 9097, pp. 485-500, Stockholm.

Drews, P.; Schirmer, I. (2014) "From Enterprise Architecture to Business Ecosystem Architecture", In International Enterprise Distributed Object Computing Conference, pp. 13-22, Ulm.

El País (2016) "Eduardo Cunha é preso em Brasília pela Lava Jato e levado para Curitiba", http://brasil.elpais.com/brasil/2016/10/19/politica/1476892544_997459

Estadão (2017) "PF indicia Eike Batista por corrupção, lavagem e organização criminosa", http://politica.estadao.com.br/noticias/geral,pf-indicia-eike-batista-porcorrupcao-lavagem-e-organizacao-criminosa,70001657686

Fusco, J. P. (2005) "Redes produtivas e cadeias de fornecimento". São Paulo: Editora Arte e Ciência.

G1 (2016) "Ex-governador Sérgio Cabral é preso pela PF na Zona Sul do Rio", http://g1.globo.com/rio-de-janeiro/noticia/2016/11/ex-governador-sergio-cabral-epreso-pela-pf-na-zona-sul-do-rio.html

Gazeta do Povo (2017) "Estudante detectou sozinha desvio milionário de bolsas que a UFPR não viu”, http://www.gazetadopovo.com.br/vida-e-cidadania/estudantedetectou-sozinha-desvio-milionario-de-bolsas-que-a-ufpr-nao-viu$52 \mathrm{c} 7 \mathrm{c} 52 \times 8961$ i4rb2qkrjeona

Hsu, C.; Lin, Y-T.; Wang, T. (2015) "A legitimacy challenge of a cross-cultural interorganizational information system", European Journal of Information Systems, v. 24, pp. 278-294. 
Kauremaa, J.; Tanskanen, K. (2016) "Designing interorganizational information systems for supply chain integration: a framework", The International Journal of Logistics Management, v. 27, n. 1, pp. 71-94.

Khalfallah, M.; Figay, N.; Ghodous, P.; da Silva, C.F. (2013) "Cross-Organizational Business Processes Modeling Using Design-by-Contract Approach”, In International IFIP Working Conference on Enterprise Interoperability, LNBIP 144, pp. 77-90, Enschede.

Lawall, A.; Schaller, T.; Reichelt, D. (2014) "Restricted Relations between Organizations for Cross-Organizational Processes", In Conference on Business Informatics, Lisbon.

Mueller, T.; Schuldt, D.; Sewald, B.; Morisse, M.; Petrikina, J. (2013) "Towards interorganizational Enterprise Architecture Management", In Americas Conference on Information Systems, Chicago.

Nunes, V.; Cappelli, C.; Costa, M. V. (2016) "Promoting Transparency in Government through FACIN: The Brazilian Government Enterprise Architecture Framework", In Workshop de Transparência em Sistemas, Rio de Janeiro.

Pedroso, J. P. (2009) "A gestão dos relacionamentos interorganizacionais cooperativos: Um estudo de caso", Dissertação (Mestrado em Administração) - Universidade Federal do Paraná, Curitiba.

Polícia Federal (2017) “Operação Lava-Jato”, http://www.pf.gov.br/imprensa/lava-jato

Ranaei, H.; Zareei, A.; Alikhani, F. (2010) "Inter-organizational Relationship Management: A Theoretical Model", International Bulletin of Business Administration, n. 9, pp. 20-30.

Sebu, M.; Ciocârlie, H. (2015) "Merging business processes for a common workflow in an organizational collaborative scenario", In International Conference on System Theory, Control and Computing, pp. 134-139, Cheile Gradistei.

Sun, K.; Yu, K. (2011) "Research on project management for Inter-Organizational Information Systems", In International Conference on E-Business and EGovernment, pp. 1-4, Shanghai.

Transparency International (2017) "Corruption perception index", https://www.transparency.org/news/feature/corruption_perceptions_index_2016

Van Fenema, P.; Keers, B.; Zijm, H. (2014) "Interorganizational Shared Services: Creating Value across Organizational Boundaries", Shared Services as a New Organizational Form, v. 13, pp. 175-217.

Zhu, Z.; Huang, H. (2007) "The Cultural Integration in the Process of Cross-border Mergers and Acquisitions", International Management Review, v. 3, n. 2, pp. 40-44. 